\title{
Selective Lengthening of the Cell Cycle in the Neurogenic Subpopulation of Neural Progenitor Cells during Mouse Brain Development
}

\author{
Federico Calegari, ${ }^{\star}$ Wulf Haubensak, ${ }^{\star}$ Christiane Haffner, and Wieland B. Huttner \\ Max Planck Institute of Molecular Cell Biology and Genetics, D-01307 Dresden, Germany
}

\begin{abstract}
During embryonic development of the mammalian brain, the average cell-cycle length of progenitor cells in the ventricular zone is known to increase. However, for any given region of the developing cortex and stage of neurogenesis, the length of the cell cycle is thought to be similar in the two coexisting subpopulations of progenitors [i.e., those undergoing (symmetric) proliferative divisions and those undergoing (either asymmetric or symmetric) neuron-generating divisions]. Using cumulative bromodeoxyuridine labeling of Tis21-green fluorescent protein knock-in mouse embryos, in which these two subpopulations of progenitors can be distinguished in vivo, we now show that at the onset as well as advanced stages of telencephalic neurogenesis, progenitors undergoing neuron-generating divisions are characterized by a significantly longer cell cycle than progenitors undergoing proliferative divisions. In addition, we find that the recently characterized neuronal progenitors dividing at the basal side of the ventricular zone and in the subventricular zone have a longer $G_{2}$ phase than those dividing at the ventricular surface. These findings are consistent with the hypothesis (Calegari and Huttner, 2003) that cell-cycle lengthening can causally contribute to neural progenitors switching from proliferative to neuron-generating divisions and may have important implications for the expansion of somatic stem cells in general.
\end{abstract}

Key words: neurogenesis; neuroepithelial cells; basal progenitors; cell cycle; Tis21; BrdU

\section{Introduction}

With the onset of neurogenesis in the embryonic mouse brain, an increasing proportion of neuroepithelial (NE) cells switch from proliferative to neuron-generating divisions (Caviness et al., 1995; McConnell, 1995; Rakic, 1995) and transform into radial glial (RG) cells (Kriegstein and Götz, 2003). As a corollary, during neurogenesis, a variable proportion of proliferating and neurogenic NE/RG cells coexist within the same area of the neuroepithelium. Also, with the onset and progression of neocortical neurogenesis, the length of the cell cycle of NE/RG cells (determined without distinguishing between proliferating and neurogenic cells), including that of neural stem cells, increases, in particular its $\mathrm{G}_{1}$ phase (Takahashi et al., 1995a; Martens et al., 2000). However, for any given region of the developing cortex and stage of neurogenesis, the cell-cycle length of NE/RG cells is thought to be

\footnotetext{
Received 0ct. 11, 2004; revised May 23, 2005; accepted May 25, 2005.

W.B.H. was supported by grants from the Deutsche Forschungsgemeinschaft (SPP 1109, Hu 275/7-2; SPP 1111, Hu 275/8-2; SFB/TR 13, B1) and the Fonds der Chemischen Industrie. Animal experiments were approved by the local authorities (license number 24-9168.11-9-2004-2). We thank Alessio Attardo for providing some immunostained cryosections, Anna Grzyb for help with confocal microscopy, the animal facility of Max Planck Institute for Molecular Cell Biology and Genetics for mouse husbandry, Dr. E. Tanaka for providing anti-BrdU antibody, and Dr. R. S. Nowakowski for kindly sending us an Excel spreadsheet to calculate cell-cycle parameters by nonlinear fit.

*F.C. and W.H. contributed equally to this work.

Correspondence should be addressed to Dr. Wieland B. Huttner, Max Planck Institute of Molecular Cell Biology and Genetics, Pfotenhauerstrasse 108, D-01307 Dresden, Germany. E-mail: huttner@mpi-cbg.de.

W. Haubensak's present address: California Institute of Technology, 1200 East California Boulevard, Pasadena, CA 91125.

DOI:10.1523/JNEUROSCI.0778-05.2005

Copyright $\odot 2005$ Society for Neuroscience $\quad$ 0270-6474/05/256533-06\$15.00/0
}

homogeneous (Cai et al., 1997). This would imply that NE/RG cells undergoing proliferative division and those undergoing neuron-generating divisions have very similar cell-cycle length.

Indeed, on the one hand, no differences in cell-cycle length have been detected when the same neocortical region was analyzed with regard to ventricular zone (VZ) versus subventricular zone (SVZ) (Reznikov and van der Kooy, 1995), although these zones have been shown recently to contain distinct neuronal progenitors (Haubensak et al., 2004; Miyata et al., 2004; Noctor et al., 2004). On the other hand, differences in bromodeoxyuridine (BrdU) incorporation during pulse labeling between distinct subpopulations of RG cells have been reported (Hartfuss et al., 2001). Thus, it cannot be excluded that the homogeneity in $\mathrm{NE} / \mathrm{RG}$ cell-cycle length at any given stage of neurogenesis (Cai et al., 1997) is more apparent than real, reflecting the lack of markers that would allow one to distinguish between distinct subpopulations of NE/RG cells, such as those undergoing proliferative versus neuron-generating divisions.

We explored previously whether the lengthening of the NE/RG cell cycle may causally contribute to NE cells switching from proliferative to neuron-generating divisions (Calegari and Huttner, 2003). Indeed, lengthening of the NE cell cycle in intact mouse embryos by the specific cyclin-dependent kinase inhibitor olomoucine was found to be sufficient to trigger premature neurogenesis in the telencephalon (Calegari and Huttner, 2003). Given these observations, and consistent with the effects of growth factors on cell-cycle regulators and NE/RG cell-cycle kinetics in cell culture (Lukaszewicz et al., 2002), we proposed a 
hypothesis explaining how the lengthening of the NE/RG cell cycle can causally contribute to NE cells switching from proliferative to neuron-generating divisions (Calegari and Huttner, 2003). This cell-cycle length hypothesis implies that NE/RG cells undergoing neuron-generating divisions should have a longer cell cycle than proliferating ones.

A powerful tool for investigating potential differences in cellcycle length between proliferating and neurogenic NE/RG cells is the recently described Tis21-green fluorescent protein (Tis21GFP) knock-in mouse line (Haubensak et al., 2004). Here, we determined cell-cycle parameters for NE/RG cells undergoing proliferative versus neuron-generating divisions, and for neurogenic progenitors dividing in the VZ versus SVZ, in Tis21-GFP knock-in mouse embryos using cumulative BrdU labeling (Nowakowski et al., 1989).

\section{Materials and Methods}

Cumulative BrdU labeling. Cumulative BrdU labeling of embryonic day 10.5 (E10.5) and E14.5 heterozygous Tis21-GFP mouse embryos (C57BL/6 background) (Haubensak et al., 2004) was performed by repeated intraperitoneal injections ( $2-4 \mathrm{~h}$ intervals; $0.31-1.25 \mathrm{mg}$ of BrdU) into pregnant mice $(20-30 \mathrm{~g})$. For details, see supplemental material (available at www.jneurosci.org).

Light microscopy. E10.5 embryos and E14.5 brains were fixed, and immunohistochemistry was performed on $10 \mu \mathrm{m}$ cryosections as described previously (Calegari and Huttner, 2003), followed by counting nuclei from images obtained with a conventional fluorescence microscope (for details, see supplemental material, available at www.jneurosci.org). Essentially the same BrdU labeling index was obtained when, in the cryosection under investigation, nuclei were scored from images obtained with a confocal microscope (see supplemental material, available at www.jneurosci.org). We analyzed cells of the VZ of the E10.5 rostral telencephalon and of the E14.5 telencephalon in the rostral archicortex and periarchicortex. The basal region of the $\mathrm{VZ}$ was distinguished from the adjacent neuronal layer (E10.5) and SVZ (E14.5) (Takahashi et al., 1995b) by (1) the shape of the nuclei (elongated ellipsoid rather than nearly circular), (2) their orientation (long axis vertical rather than oblique or horizontal), and (3) the proportion of nuclei that were GFP+ (at most one-half rather than the vast majority) (see Fig. $1 A, C-C^{\prime \prime}$ ) (Haubensak et al., 2004). Mitotic neurogenic progenitors in the basal VZ and SVZ were identified by 4',6-diamidino-2-phenylindole (DAPI) and phosphohistone $\mathrm{H} 3$ staining as well as expression of GFP.

Measurement of the cell cycle of NE/RG cells and statistical analyses. Nuclei in the VZ (identified by DAPI staining) were first classified as $\mathrm{GFP}-$ or GFP + the proportion of BrdU + nuclei in each subpopulation was determined, and cell-cycle parameters were calculated (Nowakowski et al., 1989) from the mean BrdU labeling index (three independent litters; for each litter, summing up the numbers for at least two embryos and at least two nonconsecutive cryosections per embryo). For details, see supplemental material (available at www.jneurosci.org).

\section{Results}

\section{The model system}

To investigate potential differences in cell-cycle length between NE/RG cells undergoing proliferative division and those undergoing neuron-generating divisions, we used heterozygous Tis21GFP knock-in mouse embryos, in which nuclear GFP is selectively expressed in the neurogenic, but not the proliferating, subpopulations of NE/RG cells (Haubensak et al., 2004). Tis21 is an immediate early gene (Tirone, 2001). Accordingly, in NE cells, the Tis 21 mRNA is synthesized in $G_{1}$ and degraded at the $G_{1}-S$ transition, with the Tis 21 protein persisting through $S, G_{2}$, and $M$ phases (Iacopetti et al., 1999). Consistent with this, in Tis21-GFP knock-in mouse embryos, GFP fluorescence in NE cell nuclei increases during $G_{1}$ (when the nucleus migrates from the apical surface to the basal boundary of the VZ), persists through $\mathrm{S}$ and
$\mathrm{G}_{2}$ (when the nucleus migrates back to the apical surface of the $\mathrm{VZ}$ ), and the GFP after M phase is inherited by default by the $\mathrm{NE} / \mathrm{RG}$ daughter cell as well as the newborn neuron, followed by reinduction of Tis21-GFP fluorescence in the former and its loss in the latter cell (Haubensak et al., 2004). Thus, in Tis21-GFP knock-in mouse embryos, the two subpopulations of proliferating and neurogenic progenitors can be distinguished before their entry into S phase, allowing a comparative analysis of cell-cycle length using indicators of DNA synthesis such as BrdU (see also the model in Fig. 3).

Heterozygous Tis21-GFP knock-in mouse embryos are phenotypically wild type, in line with the previously reported observations that even homozygous embryos and adult mice do not show any obvious phenotype (Haubensak et al., 2004). After cumulative BrdU labeling, we analyzed BrdU incorporation into GFP + and GFP - nuclei in the VZ in those regions of the developing forebrain for which a mean cell-cycle length has been reported previously (i.e., in the E10.5 rostral telencephalon and the E14.5 rostral archicortex and periarchicortex) (Takahashi et al., 1995a; Cai et al., 1997). As shown in Figure $1, A$ and $C-C^{\prime \prime}$, four distinct subpopulations of nuclei in the VZ could be distinguished: (1) GFP-/BrdU- (white arrowheads), (2) GFP-/ $\mathrm{BrdU}+$ (red arrowheads), (3) GFP $+/ \mathrm{BrdU}-$ (green arrowheads), and (4) GFP+/BrdU+ (green/red arrowheads). This demonstrates that the rate of S phase entry of NE/RG cells over time, and thus cell-cycle length, can be determined separately for the two subpopulations of proliferating $(\mathrm{GFP}-)$ and neurogenic $(\mathrm{GFP}+)$ progenitor cells (see also the model in Fig. 3).

\section{At the onset of neurogenesis, NE cells switching from proliferative to neuron-generating divisions lengthen their cell cycle}

Cumulative BrdU labeling of E10.5 Tis21-GFP mouse embryos (i.e., at the onset of telencephalic neurogenesis) revealed that $\mathrm{NE}$ cells that will undergo neuron-generating divisions $(\mathrm{GFP}+)$ show a $20 \%$ increase in cell-cycle length compared with NE cells that will undergo proliferative divisions (GFP-) (Fig. $1 B$ ). Calculations according to Nowakowski et al. (1989) yielded a cellcycle length of 13.0 and $10.9 \mathrm{~h}$, respectively (Table 1 ). The longer cell cycle of the neurogenic (GFP+) subpopulation was attributable to a lengthening specifically of the $G_{1}$ phase (Table 1 ), which is in agreement with the previous finding that the overall lengthening of the NE/RG cell cycle during neurogenesis reflects a selective increase in $\mathrm{G}_{1}$ (Takahashi et al., 1995a). It should be noted, and will be discussed below, that the fraction of dividing cells, also referred to as growth fraction (Nowakowski et al., 1989) (Fig. $1 B$, dashed lines), was as high for the GFP + VZ cells $(85.6 \pm 8.1 \%)$ as for the GFP - VZ cells $(83.2 \pm 3.0 \%)$ (Table 1$)$.

It could be argued that the determination of cell-cycle length by cumulative BrdU labeling leads, in the case of the GFP+ VZ cells, to an overestimation because of the presence of newborn postmitotic neurons in the VZ that are GFP+ (Haubensak et al., 2004) but BrdU - and that are replaced by GFP+/BrdU+ neurons only during the last hours of BrdU labeling. However, this is not the case for the following reasons. First, even in the E11.5 telencephalon (i.e., a stage corresponding to the end of the cumulative BrdU labeling in Fig. $1 B$ ), neurons (identified by $\beta$-IIItubulin immunoreactivity) constitute only $7 \pm 3 \%$ of the GFP + $\mathrm{VZ}$ cells (data not shown). Second, neurons generated by asymmetric division of GFP + NE cells at the apical side of the neuroepithelium have left the VZ by the time their sister NE cells reach the basal side of the VZ for S phase (Haubensak et al., 2004) and therefore do not cause a reduction of the BrdU labeling index of 

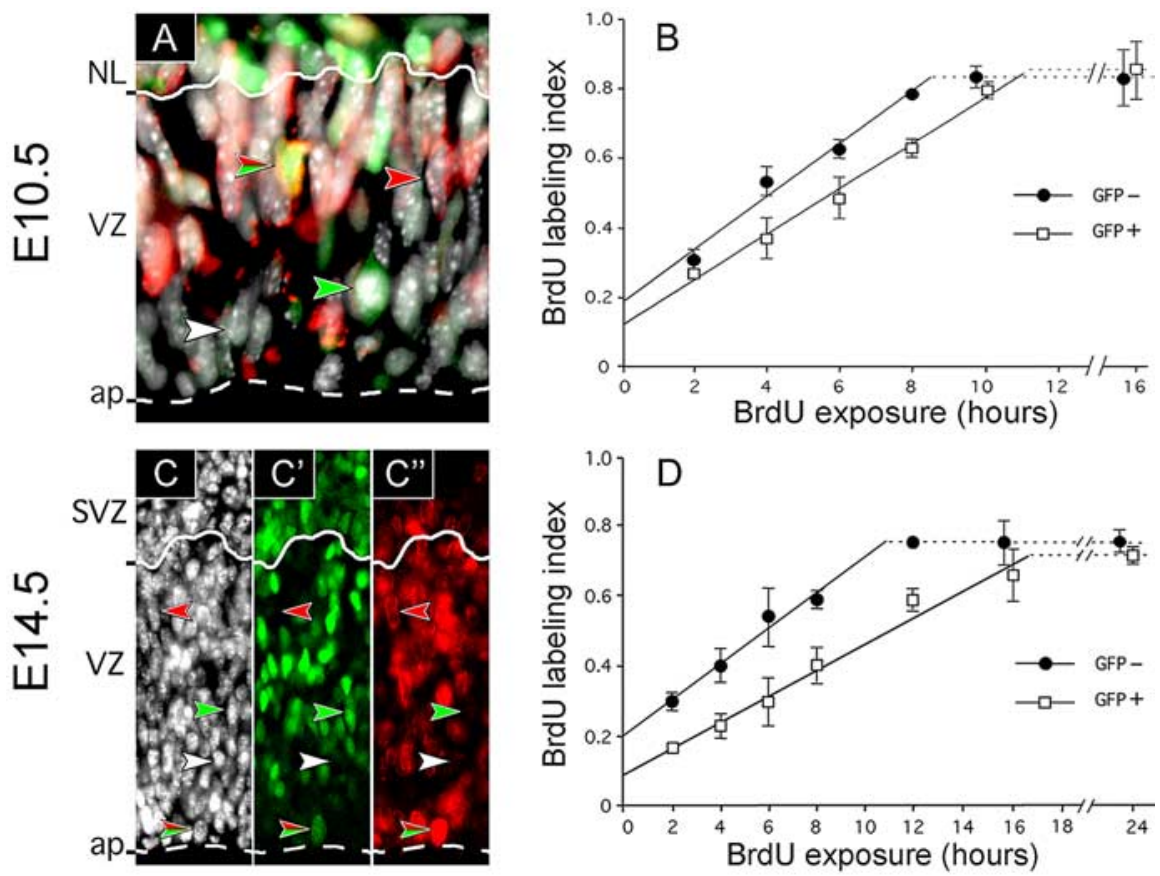

Figure 1. Cumulative BrdU labeling of NE/RG cells in E10.5 and E14.5 heterozygous Tis21-GFP embryos. $A, C-C^{\prime \prime}$, Cells in the telencephalic neuroepithelium of an E10.5 $(\boldsymbol{A})$ and E14.5 ( $\left.\boldsymbol{C}-\boldsymbol{C}^{\prime \prime}\right)$ heterozygous Tis21-GFP embryo stained for DNA (white; $\boldsymbol{A}, \boldsymbol{C}$ ) and immunostained for GFP ( green; $A, C^{\prime}$ ) and BrdU ( $4 \mathrm{~h}$ labeling; red; $A, C^{\prime \prime}$ ). Note the examples of the four types of stained nuclei, (1) GFP - /BrdU - (white arrowheads), (2) GFP +/BrdU - (green arrowheads), (3) GFP - /BrdU + (red arrowheads), and (4) $\mathrm{GFP}+/ \mathbf{B r d U}+$ (green/red arrowheads), in the merged image $(\boldsymbol{A})$ and the three individual channels $\left(\boldsymbol{C}-\boldsymbol{C}^{\prime \prime}\right)$. Solid and dashed lines represent the basal boundary of VZ to the neuronal layer (NL) (A) and SVZ (C-C') and the apical (ap) surface, respectively. $\boldsymbol{B}, \boldsymbol{D}$, Cumulative BrdU labeling of GFP - (filled circles) and GFP + (open squares) nuclei, identified as in $\boldsymbol{A}$ and $\mathbf{C}-\boldsymbol{C}^{\prime}$, in VZ cells at E10.5 $(\boldsymbol{B})$ and E14.5 (D); all nuclei stained had a BrdU labeling index 1.0. Data are the mean of three (E14.5; $12 \mathrm{~h} ; 2$ litters) independent litters; bars indicate SD (E14.5; $12 \mathrm{~h}$; variation of the 2 litters from the mean). Horizontal dashed lines represent the growth fraction.

the GFP + population (see also the model in Fig. 3). It follows that the difference in cumulative BrdU labeling observed between $\mathrm{GFP}-$ and GFP + VZ cells (Fig. $1 \mathrm{~B}$ ) reflects a genuine difference in NE cell-cycle length.

\section{Additional lengthening of the cell cycle in the neurogenic subpopulation of neuronal progenitor cells at advanced stages of neurogenesis}

The previously reported increase in the mean cell-cycle length of $\mathrm{NE} / \mathrm{RG}$ cells in correlation with the progression of neurogenesis (Takahashi et al., 1995a) could partly be explained by the rise in the proportion of the more slowly cycling, GFP + NE/RG cells (Fig. $1 \mathrm{~B}$ ) during development from $5 \%$ at E10.5 to $50 \%$ at E14.5 (Haubensak et al., 2004) (Table 1). In addition, this increase could be because of (1) an additional lengthening of the cell cycle selectively in the GFP+ subpopulation or (2) an additional lengthening in both GFP + and GFP - subpopulations. Cumulative BrdU labeling of E14.5 Tis21-GFP mouse embryos (Fig. 1D) showed that the latter is the case, with the GFP - NE/RG subpopulation increasing their cell-cycle length from E10.5 to E14.5 by $36 \%$ to $14.8 \mathrm{~h}$ and the GFP + NE/RG subpopulation by $47 \%$ to $19.1 \mathrm{~h}$ (Table 1). Thus, neurogenic NE/RG cells at this advanced stage of neurogenesis show a substantial increase (30\%) in cellcycle length compared with proliferating ones. (For the significance of the cell-cycle lengthening in the GFP- NE/RG cell subpopulation from E10.5 to E14.5, see Discussion.) As was the case at E10.5, $G_{1}$ was the only phase of the NE/RG cell cycle that lengthened (1) in E14.5 neurogenic (GFP+) cells compared with E14.5 proliferating (GFP-) cells and (2) in E14.5 compared with
E10.5 GFP + cells and E14.5 compared with E10.5 GFP - cells (Table 1). Considering that at this stage $50 \%$ of NE/RG cells are GFP+ (Haubensak et al., 2004), the mean value of the cell-cycle length of all NE/RG cells would be $17.4 \mathrm{~h}$ (Table 1 ), which is in good agreement with previous data (Takahashi et al., 1995a).

As was the case at E10.5, the growth fraction at E14.5 (Fig. 1D, dashed lines) was essentially as high for the GFP+ NE/RG cells $(70.7 \pm 2.4 \%)$ as for the GFP - cells (74.8 $\pm 3.1 \%)$ (Table 1$)$, indicating that the switch of NE/RG cells from proliferation $(\mathrm{GFP}-)$ to neurogenesis $(\mathrm{GFP}+)$ is not accompanied by an increase in the proportion of postmitotic cells in the VZ. This in turn eliminates the possibility that, in the case of the GFP+ VZ cells, cell-cycle length was overestimated because of the transient presence of newborn postmitotic neurons in the VZ.

\section{Difference in cell-cycle parameters between neurogenic NE/RG cells and basal progenitors}

Neurons in the telencephalon originate not only from NE/RG cells dividing asymmetrically at the apical surface of the VZ (generating one neuron plus one NE/RG cell) but also from progenitors dividing symmetrically at the basal side of the VZ and in the SVZ (generating two neurons) (Haubensak et al., 2004; Miyata et al., 2004; Noctor et al., 2004). It was therefore of interest to investigate whether these two subtypes of neuronal progenitors have the same or a different cell-cycle length.

At E10.5, apically dividing GFP + NE cells are far more abundant than GFP + basal progenitors (Haubensak et al., 2004), and hence the $20 \%$ increase in cell-cycle length in the GFP+, compared with the GFP-, VZ cells at this stage (Fig. $1 B$ ) primarily reflects the difference between apically dividing neurogenic and proliferating NE cells. In contrast, at later stages of neurogenesis, GFP + basal progenitors outnumber apically dividing GFP+ NE/RG cells (Haubensak et al., 2004), and hence the greater, 30\% increase in cell-cycle length in GFP+ VZ cells at E14.5 (Fig. 1D) raises the possibility that the GFP + basal neuronal progenitors have an even slower cell cycle than the apically dividing neurogenic $(\mathrm{GFP}+) \mathrm{NE} / \mathrm{RG}$ cells. An established criterion for distinguishing between these two subpopulations of neuronal progenitors is their different sites of mitosis (Haubensak et al., 2004). Because the GFP + basal progenitors divide into two postmitotic cells (neurons) (Haubensak et al., 2004; Noctor et al., 2004), analysis of their cell cycle by cumulative BrdU labeling is restricted to the interval between $S$ phase and mitosis (see also the model in Fig. 3).

Cumulative BrdU labeling of E14.5 Tis21-GFP mouse embryos for $4 \mathrm{~h}$ followed by analysis of GFP + cells in mitosis (Fig. 2) showed that only one-half ( $52 \%$ ) of the mitotic neuronal progenitors in the SVZ (Fig. $2 f$ ), but the vast majority $(82 \%)$ of the mitotic neurogenic NE/RG cells at the apical surface of the VZ (Fig. 2l), had BrdU-labeled chromosomes. In addition, the apparent amount of BrdU incorporation after $4 \mathrm{~h}$ of labeling was, in 
Table 1. Proportion and cell-cycle parameters of GFP - and GFP + NE/RG cells

\begin{tabular}{|c|c|c|c|c|c|c|c|c|c|}
\hline \multirow[b]{2}{*}{ Stage } & \multirow[b]{2}{*}{ VZ cells } & \multicolumn{2}{|c|}{ Proportion $^{a}(\%)$} & \multicolumn{5}{|c|}{ Cell-cycle parameters $^{b}$ (hours) } & \multirow[b]{2}{*}{$(\% \pm \mathrm{SD}) \mathrm{GF}$} \\
\hline & & $t_{0 \mathrm{~h}}$ & $t_{24 \mathrm{~h}}$ & $T_{\mathrm{C}}-T_{\mathrm{S}}$ & $T_{\mathrm{S}}$ & $T_{\mathrm{C}}$ & $T_{\mathrm{G}_{2}+\mathrm{M}}$ & $T_{\mathrm{G}_{1}}$ & \\
\hline \multirow[t]{3}{*}{$\mathrm{E} 10.5$} & GFP- & 95 & 90 & 8.5 & 2.4 & 10.9 & $<2.0$ & $>6.5$ & $83 \pm 3$ \\
\hline & $\mathrm{GFP}+$ & 5 & 10 & 11.3 & $1.7^{c}$ & 13.0 & $<2.0$ & $>9.3$ & $86 \pm 8$ \\
\hline & Mean & & & 8.8 & 2.3 & 11.1 & $<2.0$ & $>6.8$ & $85 \pm 4$ \\
\hline \multirow[t]{3}{*}{ E14.5 } & GFP- & 50 & 40 & 10.9 & 3.9 & 14.8 & $<2.0$ & $>8.9$ & $75 \pm 3$ \\
\hline & $\mathrm{GFP}+$ & 50 & 60 & 16.7 & $2.4^{c}$ & 19.1 & $<2.0$ & $>14.7$ & $71 \pm 2$ \\
\hline & Mean & & & 14.4 & 3.0 & 17.4 & $<2.0$ & $>12.4$ & $73 \pm 2$ \\
\hline
\end{tabular}

Mean values of cell-cycle parameters for the sum of GFP - and GFP $+\mathrm{NE} / \mathrm{RG}$ cells were calculated using their proportion at $\mathrm{E} 11.5$ and $\mathrm{E} 15.5\left(t_{24} \mathrm{~h}\right) . \mathrm{GF}$, Growth fraction; $T_{C}$, total length of the cell cycle; $T_{\mathrm{S}}$, length of the $S$ phase; $T_{\mathrm{G}}+\mathrm{M}_{\mathrm{M}}$, length of the $G_{2}$ phase plus the $M$ phase; $T_{G_{1}}$, length of the $G_{1}$ phase.

${ }^{a}$ Data from Haubensak et al. (2004).

${ }^{b}$ Calculated according to Nowakowski et al. (1989). $T_{\mathrm{G}_{2}}+\mathrm{M}$ is only given for the apically dividing NE/RG cells.

' Values are underestimations (see supplemental material, available at www.jneurosci.org).
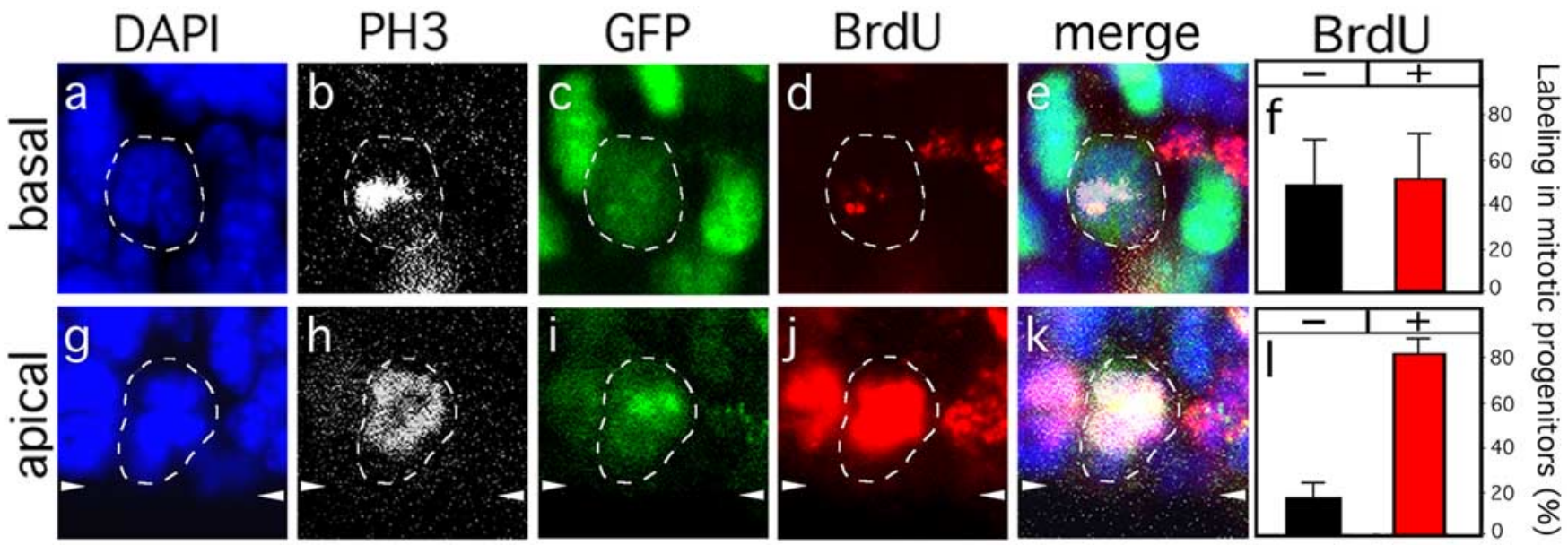

Figure 2. BrdU labeling in mitotic neuronal progenitors. Dashed lines indicate mitotic basal $(\boldsymbol{a}-\boldsymbol{e})$ and apical $(\boldsymbol{g}-\boldsymbol{k})$ progenitors in the telencephalic SVZ (basal) and VZ (apical), respectively, of an E14.5 Tis21-GFP heterozygous embryo exposed to BrdU for $4 \mathrm{~h}$. Mitotic cells were identified by DAPI ( $\boldsymbol{a}, \boldsymbol{g}$; blue) and anti-phosphohistone H3 (PH3; $\boldsymbol{b}, \boldsymbol{h}$; white) staining. Only GFP + (c, $\boldsymbol{i} ;$ green) mitotic progenitors were analyzed for incorporation of BrdU $(\boldsymbol{d}, \boldsymbol{j}$; red). White arrowheads at the margins of $\boldsymbol{g}-\boldsymbol{k}$ indicate the apical surface of the VZ. $\boldsymbol{f}, \boldsymbol{I}$, Percentage of BrdU $-($ black bars) and BrdU + (red bars) mitotic GFP + basal $(\boldsymbol{f})$ and apical $(\boldsymbol{I})$ neuronal progenitors [5 embryos from 3 litters; basal progenitors, $n=21$; apical progenitors, $n=33$; each randomly distributed into 3 groups for SD (bars); basal BrdU + percentage vs apical BrdU + percentage; $p<0.05]$.

most cases, clearly less for the mitotic GFP + SVZ cells (Fig. 2d) than for the apical mitotic GFP+ VZ cells (Fig. 2j). After $6 \mathrm{~h}$ or more of cumulative BrdU labeling, virtually all mitotic GFP+ cells in the SVZ, like those at the apical surface of the VZ, showed robust BrdU labeling (data not shown). Together, these data demonstrate that the $G_{2}$ phase of basal, symmetrically dividing, neuronal progenitors is longer $(>4 \mathrm{~h})$ than that of the apical, asymmetrically dividing, neurogenic NE/RG cells ( $\leq 4 \mathrm{~h}$ ). In fact, regarding the latter cells, already after $2 \mathrm{~h}$ of BrdU labeling, $>90 \%$ of the apical mitotic GFP + as well as the apical mitotic GFPNE/RG cells had BrdU-labeled chromosomes. This indicates that the $G_{2}$ phase of both the neurogenic and proliferating subpopulations of NE/RG cells is $<2 \mathrm{~h}$ (Table 1 ), consistent with previous observations (Takahashi et al., 1995a).

\section{Discussion}

We have determined cell-cycle parameters in three coexisting subpopulations of neural progenitors (i.e., NE/RG cells undergoing either symmetric proliferative or asymmetric neurongenerating divisions and basal progenitors undergoing symmetric neuron-generating division) (Fig. 3). These experiments make three major points. First, NE/RG cells undergoing neurongenerating division $(\mathrm{GFP}+)$ have a longer cell cycle than those undergoing proliferative division $(\mathrm{GFP}-)$ (Fig. 1). This increase in NE/RG cell-cycle length mostly concerns $G_{1}$, because it cannot be explained by the differences in the length of $S$ (apparent values at E10.5, GFP-, $2.4 \mathrm{~h}, \mathrm{GFP}+, 1.7 \mathrm{~h}$; at E14.5, GFP-, $3.9 \mathrm{~h}$, $\mathrm{GFP}+, 2.4 \mathrm{~h}$ ) and because we did not detect major differences between GFP - and GFP + NE/RG cells with regard to the length of $\mathrm{G}_{2} / \mathrm{M}$ ( $\leq 2 \mathrm{~h}$ for both). Given that (1) proliferating and neurogenic NE/RG cells differ by the absence and presence of Tis 21 (and Tis21-GFP) expression (Iacopetti et al., 1999; Haubensak et al., 2004), respectively, and (2) Tis 21 has been shown to inhibit $\mathrm{G}_{1}$ progression in other cells (Tirone, 2001), Tis21 is a likely candidate to be one (although clearly not the only) factor contributing to the lengthening of the NE/RG cell cycle observed during neurogenesis (Malatesta et al., 2000).

Second, the switch of NE/RG cells from proliferation (GFP-) to neurogenesis $(\mathrm{GFP}+)$ is not associated with an increase in postmitotic cells in the VZ (as we observed essentially the same growth fraction for both subpopulations) (Fig. 1). In other words, Tis21 expression does not cause NE/RG cells to become postmitotic. This implies that neurons (which initially are GFP+) (Haubensak et al., 2004) do not arise by NE/RG cells first becoming postmitotic and then transforming into neurons, but rather from mitotic Tis21-expressing NE/RG cells. Together, commitment of NE/RG cells to neurogenesis (Tis21 expression) 


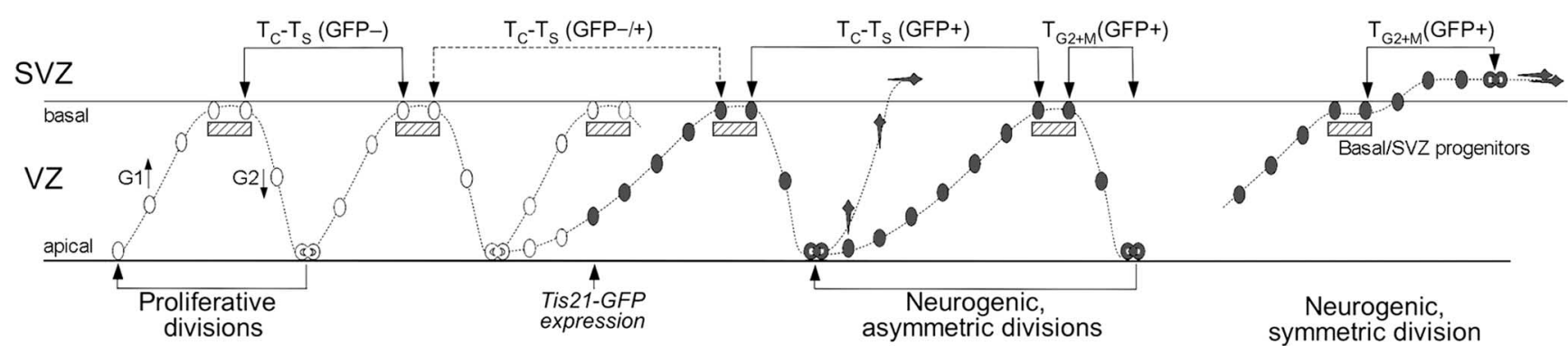

Figure 3. Relationship between interkinetic nuclear migration, Tis21-GFP expression, and BrdU incorporation of NE/RG cells and basal/SVZ progenitors. White nuclei, GFP-NE cells undergoing proliferative divisions; dark gray nuclei, GFP + NE/RG cells and basal/SVZ progenitors undergoing neurogenic divisions; hatched boxes, S phase nuclei incorporating BrdU; dark gray polygons, neurons. Lines flanked by arrows indicate the length of $T_{\mathrm{C}}-T_{\mathrm{S}}$ (the total length of the cell cycle minus the $S$ phase) for GFP - versus GFP $+N E / R G$ cells and of $T_{\mathrm{G}_{2}+\mathrm{M}}$ (the $\mathrm{G}_{2}$ phase plus the minimum period at the end of the $S$ phase necessary to incorporate detectable amounts of BrdU plus the fraction of $\mathrm{M}$ phase needed to identify condensed chromatids or detect anti-phosphohistone $\mathrm{H} 3$ immunoreactivity) for GFP $+\mathrm{NE} / \mathrm{RG}$ cells versus GFP + basal/SVZ progenitors. The slope of the apical-to-basal migration of nuclei indicates the difference in the length of $\mathrm{G}_{1}$. For details, see text and supplemental material (available at www.jneurosci.org).

is accompanied by a lengthening, but not a block, of their cell cycle.

Third, extending previous studies on differences in cell-cycle parameters between VZ and SVZ progenitors (Takahashi et al., 1995b), basal neurogenic progenitors were found to have a longer $\mathrm{G}_{2}$ than the neurogenic subpopulation of the apically dividing NE/RG cells (Figs. 2, 3). The significance of this $\mathrm{G}_{2}$ lengthening is not yet known. However, one may speculate that it reflects the retraction of the apical process of NE/RG cells (including the loss of their adherens junctions) as they transform into basal progenitors, which can occur after the nucleus has reached the basal side of the VZ (Miyata et al., 2004) and may be required for basal progenitors to divide into two neurons.

In light of the promotion of neurogenesis by molecules that have been shown, or appear, to lengthen the cell cycle of NE/RG cells (Malatesta et al., 2000; Lukaszewicz et al., 2002; Calegari and Huttner, 2003; Canzoniere et al., 2004), we proposed previously that the lengthening of the cell cycle can causally contribute to neural progenitor cells switching from proliferative to neurongenerating divisions (Calegari and Huttner, 2003). In this cellcycle length model, a cell fate determinant may or may not cause a cell fate change, depending on whether the length of time this determinant can act is sufficient. The present data, showing that neurogenic NE/RG cells have a longer cell cycle than proliferating ones (Fig. 1), support the cell-cycle length hypothesis (Calegari and Huttner, 2003). Moreover, with regard to asymmetric versus symmetric neurogenic divisions, the additional lengthening of the cell cycle in the GFP + subpopulation of NE/RG cells concomitant with the progression of neurogenesis (Fig. 1) would explain (Calegari and Huttner, 2003) why these neuronal progenitors undergo symmetric (rather than asymmetric) neurogenic divisions at later stages of neurogenesis (McConnell, 1995).

The observation that the cell cycle also lengthened, from E10.5 to E14.5, in the GFP - subpopulation of NE/RG cells does not contradict the cell-cycle length hypothesis but is, in fact, what would be expected, for the following reason. Although at E10.5, most, if not all, GFP - NE cells undergo symmetric proliferative division, the GFP - NE/RG cells at E14.5 not only generate more $\mathrm{NE} / \mathrm{RG}$ cells but also divide to generate more differentiated cells such as those of the glial lineage (Parnavelas, 1999) and basal progenitors (Haubensak et al., 2004).

Likewise, the finding that, in terms of absolute time, the cell cycle was longer in E14.5 GFP - cells than in E10.5 GFP+ cells does not contradict the cell-cycle length hypothesis either. If the distribution of a key cell fate determinant during mitosis is more unequal at E14.5 than at E10.5 (say, 70:30\% rather than 60:40\%) [Calegari and Huttner (2003), their Fig. 5], our model predicts that a cell-cycle lengthening sufficient to induce a cell fate change at E10.5 will no longer be sufficient to do so at E14.5.

Importantly, our findings, together with observations on isolated neuronal progenitors in culture (Lukaszewicz et al., 2002) and transgenic mice (Hodge et al., 2004), suggest that shortening the cell cycle of neural progenitor cells should prevent their differentiation. This may be a crucial aspect for somatic stem cells in general, which have been more difficult to expand than embryonic stem cells. It could be significant that embryonic stem cells have a remarkably short cell cycle (Burdon et al., 2002). Perhaps neural and other somatic stem cells can be expanded more efficiently if their cell cycle is accelerated.

\section{References}

Burdon T, Smith A, Savatier P (2002) Signalling, cell cycle and pluripotency in embryonic stem cells. Trends Cell Biol 12:432-438.

Cai L, Hayes NL, Nowakowski RS (1997) Local homogeneity of cell cycle length in developing mouse cortex. J Neurosci 17:2079-2087.

Calegari F, Huttner WB (2003) An inhibition of cyclin-dependent kinases that lengthens, but does not arrest, neuroepithelial cell cycle induces premature neurogenesis. J Cell Sci 116:4947-4955.

Canzoniere D, Farioli-Vecchioli S, Conti F, Ciotti MT, Tata AM, AugustiTocco G, Mattei E, Lakshmana MK, Krizhanovsky V, Reeves SA, Giovannoni R, Castano F, Servadio A, Ben-Arie N, Tirone F (2004) Dual control of neurogenesis by PC3 through cell cycle inhibition and induction of Math1. J Neurosci 24:3355-3369.

Caviness Jr VS, Takahashi T, Nowakowski RS (1995) Numbers, time and neocortical neuronogenesis: a general developmental and evolutionary model. Trends Neurosci 18:379-383.

Hartfuss E, Galli R, Heins N, Götz M (2001) Characterization of CNS precursor subtypes and radial glia. Dev Biol 229:15-30.

Haubensak W, Attardo A, Denk W, Huttner WB (2004) Neurons arise in the basal neuroepithelium of the early mammalian telencephalon: a major site of neurogenesis. Proc Natl Acad Sci USA 101:3196-3201.

Hodge RD, D'Ercole AJ, O’Kusky JR (2004) Insulin-like growth factor-I accelerates the cell cycle by decreasing $G_{1}$ phase length and increases cell cycle reentry in the embryonic cerebral cortex. J Neurosci 24:10201-10210.

Iacopetti P, Michelini M, Stuckmann I, Oback B, Aaku-Saraste E, Huttner WB (1999) Expression of the antiproliferative gene TIS21 at the onset of neurogenesis identifies single neuroepithelial cells that switch from proliferative to neuron-generating division. Proc Natl Acad Sci USA 96:4639-4644.

Kriegstein AR, Götz M (2003) Radial glia diversity: a matter of cell fate. Glia 43:37-43. 
Lukaszewicz A, Savatier P, Cortay V, Kennedy H, Dehay C (2002) Contrasting effects of basic fibroblast growth factor and neurotrophin 3 on cell cycle kinetics of mouse cortical stem cells. J Neurosci 22:6610-6622.

Malatesta P, Götz M, Barsacchi G, Price J, Zoncu R, Cremisi F (2000) PC3 overexpression affects the pattern of cell division of rat cortical precursors. Mech Dev 90:17-28.

Martens DJ, Tropepe V, van Der Kooy D (2000) Separate proliferation kinetics of fibroblast growth factor-responsive and epidermal growth factor-responsive neural stem cells within the embryonic forebrain germinal zone. J Neurosci 20:1085-1095.

McConnell SK (1995) Constructing the cerebral cortex: neurogenesis and fate determination. Neuron 15:761-768.

Miyata T, Kawaguchi A, Saito K, Kawano M, Muto T, Ogawa M (2004) Asymmetric production of surface-dividing and non-surface-dividing cortical progenitor cells. Development 131:3133-3145.

Noctor SC, Martinez-Cerdeno V, Ivic L, Kriegstein AR (2004) Cortical neurons arise in symmetric and asymmetric division zones and migrate through specific phases. Nat Neurosci 7:136-144.

Nowakowski RS, Lewin SB, Miller MW (1989) Bromodeoxyuridine immunohistochemical determination of the lengths of the cell cycle and the
DNA-synthetic phase for an anatomically defined population. J Neurocytol 18:311-318

Parnavelas JG (1999) Glial cell lineages in the rat cerebral cortex. Exp Neurol 156:418-429.

Rakic P (1995) A small step for the cell, a giant leap for mankind: a hypothesis of neocortical expansion during evolution. Trends Neurosci 18:383-388.

Reznikov K, van der Kooy D (1995) Variability and partial synchrony of the cell cycle in the germinal zone of the early embryonic cerebral cortex. J Comp Neurol 360:536-554.

Takahashi T, Nowakowski RS, Caviness Jr VS (1995a) The cell cycle of the pseudostratified ventricular epithelium of the embryonic murine cerebral wall. J Neurosci 15:6046-6057.

Takahashi T, Nowakowski RS, Caviness Jr VS (1995b) Early ontogeny of the secondary proliferative population of the embryonic murine cerebral wall. J Neurosci 15:6058-6068.

Tirone F (2001) The gene PC3(TIS21/BTG2), prototype member of the PC3/BTG/TOB family: regulator in control of cell growth, differentiation, and DNA repair? J Cell Physiol 187:155-165. 